\title{
Fuzzy Quadratic Programming Problem with Fuzzy Decision Variables: Acase of Portfolio Risk Minimization
}

Mitali Madhumita Acharya ( $\square$ mitali.acharyafpy@kiit.ac.in )

KIIT University

ADMASU TADESSE

KIITS University

Berhanu Belay

Debre Tabor University

Acharya Srikumar

KIITS University

Manoranjan Sahoo

Kalinga Institute of Industrial Technology

\section{Research Article}

Keywords: Portfolio Optimization, Quadratic Programming, fuzzy decision variable, Genetic Algorithm.

Posted Date: January 20th, 2022

DOI: https://doi.org/10.21203/rs.3.rs-1215613/v1

License: (c) (i) This work is licensed under a Creative Commons Attribution 4.0 International License. Read Full License 


\title{
Fuzzy Quadratic Programming Problem with Fuzzy Decision Variables: Acase of Portfolio Risk Minimization
}

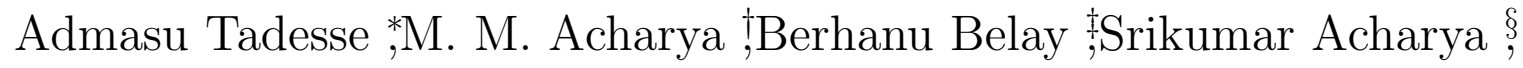 \\ Manoranjan Sahoo ? \\ Department of Mathematics, School of Applied Sciences, KIIT University, Bhubaneswar, India ${ }^{*}$, § \\ Department of Mathematics, Collage of Natural and computational Sciences, Debretabor University, Ethiopia $\dagger$
}

\begin{abstract}
This manuscript is concerned with the solution procedure of a fuzzy quadratic programming problem on risk adjusted return of portfolios by simulation-based genetic algorithm. In this manuscript, parameters of the programming problem are considered as triangular fuzzy numbers. The decision variables are assumed to be triangular fuzzy variables. In contrast to the classical technique, the proposed approach does not require the derivation of crisp equivalent form. The fuzziness of parameters and decision variables of the objective functions is first addressed using a ranking function formula implemented by MATLAB, which combines the strategy used in Genetic Algorithm. The property of fuzzy inequality constraint is used to handle the fuzziness of constraint functions. The feasibility requirements are maintained throughout the problem. A case study is used to demonstrate the proposed methodology.
\end{abstract}

\footnotetext{
*E-mail:1881140@kiit.ac.in

${ }^{\dagger}$ E-mail:mitali.acharyafpy@kiit.ac.in(Corresponding author)

${ }^{\ddagger}$ E-mail: berhanubelay2@gmail.com

$\S$ E-mail: sacharyafma@kiit.ac.in

IE-mail: mrshoofma@kiit.ac.in
} 
Keywords: Portfolio Optimization, Quadratic Programming, fuzzy decision variable, Genetic Algorithm.

\section{Introduction}

Scientifically, the decision of the decision maker is based on the accessibility of information from the mathematical modeling environment. However, the majority of the data are imprecise. The decision maker takes a reasoned decision based on the uncertainty involved in the mathematical programming problem in order to deal with imprecise parameters. Decision makers find its challenge to communicate their preferences using exact numbers due to the intricacy of the mathematical programming. Due of this issue, researchers proposed the fuzzy linear programming problem as a mathematical model for dealing with uncertainties in operations research. Bellman \& Zadeh (1970) proposed the idea of making decisions in a fuzzy environment. As a result of their findings, many scholars have developed new strategies for solving fuzzy mathematical programming problems. In this work applications of fuzzy quadratic programming(FQP) problem on optimizations of risk adjusted stock portfolio will be addressed. The mathematical programming problem of quadratic programming (QP) is defined as the optimization (minimization or maximisation) of a quadratic function subject to linear constraints (Rout et al. (2019)). Several algorithms such as (Powell $(1985)$, Lawson \& Hanson (1995), Goldfarb \& Idnani (1983)) etc., have been developed a method to solve QP

problems. Fully fuzzy multi-objective and multi-level integer QP problems were introduced by Fathy (2019), in which all the programming problem's parameters and decision variables are fuzzy numbers. Nasseri et al. (2019) suggested a fuzzy multi-objective quadratic model with variable constraints for optimal water resource allocation among different water-user sectors. The goal programming approach has been used by a number of scholars to solve fuzzy mathematical programming problem. Pramanik \& Dey (2011) proposed a fuzzy goal programming (FGP) approach to solve multi-objective quadratic programming issue based on prioritization. Mussafi \& Ismail (2020), MUSSAFI \& ISMAIL (2021) proposed a strategy for reducing the risk of a selected portfolio by implementing quadratic programming. Unlike 
most work in the applications field of QP problems, which are done in a precise setting, we have proposed our work in a fuzzy context. In this research paper, both parameters and decisions variables are considered to be triangular fuzzy number. "Many researchers have investigated on evolutionary computing and how it might be used to solve optimization problems. One such popular algorithm is GA, which is an efficient algorithm for tackling such type of problems." Gessesse et al. (2020). Genetic Algorithm (GA) is one of the heuristic algorithms used to solve optimization problems in artificial intelligence and Mathematics. GA inspired by Darwin's Theory of Evolution based on an intelligent exploitation of a random search

This paper proposes an algorithm and develops a novel technique for tackling the problem based on this concept. We employed a ranking function-based GA to solve the suggested model, where the former manages the fuzziness of the parameter-decision variables and GA solves the crisp QP programming problem. The fuzzy optimal answer is produced by solving the model obtained thus far. In contrast to a classical technique, the derivation of crisp equivalent is not require in the proposed approach. A case study has been used to demonstrate the proposed technique.

The rest of the article is organized as follows: Following the introduction, preliminaries of basic terminologies are presented in Section 2. The mathematical model is defined and discussed in Section 3. In Section 4, the GA-based ranking function approach and associated solution procedure are provided. In Section 5 and Section6 the Theoretical Case Study and simulation results respectively are discussed. In Section 7, the concluding remarks are indicated, followed by references.

\section{Basic Preliminaries}

Definition 2.1 The membership function $\left(\mu_{\widetilde{B}}(x)\right)$ of a triangular fuzzy number $\widetilde{B}=\left(b_{1}, b_{2}, b_{3}\right)$ with real numbers $b_{1}, b_{2}$ and $b_{3}$, is defined as; 
$\mu_{\tilde{B}}(x)= \begin{cases}0 & , x \leq a \\ \frac{x-b_{1}}{b_{2}-b_{1}} & , b_{1} \leq x \leq b_{2} \\ \frac{b_{3}-x}{b_{3}-b_{2}} & , b_{2} \leq x \leq b_{3} \\ 0 & , \text { otherwise }\end{cases}$

Definition 2.2 Fathy (2019) Let $\tilde{b}=\left(b_{1}, b_{2}, b_{3}\right)$ and $\widetilde{c}=\left(c_{1}, c_{2}, c_{3}\right)$ be two triangular fuzzy numbers. Then, the basic arithmetic operations will be defined as follows

$\widetilde{b}+\widetilde{c}=\left(b_{1}+c_{1}, b_{2}+c_{2}, b_{3}+c_{3}\right)$

$\widetilde{b}-\widetilde{c}=\left(b_{1}-c_{3}, b_{2}-c_{2}, b_{3}-c_{1}\right)$

$\widetilde{b} \widetilde{c}=\left(b_{1} c_{1}, b_{2} c_{2}, b_{3} c_{3}\right)$ if $b_{1} \geq 0$

$\widetilde{b} \widetilde{c}=\left(b_{1} c_{3}, b_{2} c_{2}, b_{3} c_{3}\right)$ if $b_{1} \leq 0$ and $b_{3} \geq 0$

$\widetilde{b} \widetilde{c}=\left(b_{1} c_{3}, b_{2} c_{2}, b_{3} c_{1}\right)$ if $b_{3} \geq 0 k \widetilde{b}=\left(k b_{1}, k b_{2}, k b_{3}\right)$ if $k \geq 0 k \widetilde{b}=\left(k b_{3}, k b_{2}, k b_{1}\right)$ if $k \leq 0$

Definition 2.3 "A ranking function is a function $\mathbb{R}: F(R) \rightarrow R$, where $\mathrm{F}(\mathrm{R})$ is a set of fuzzy numbers defined on set of real numbers, which maps each fuzzy number into the real line, where a natural order exists. Let $\widetilde{a}=\left(b_{1}, b_{2}, b_{3}\right)$ be a triangular fuzzy number, then $\mathbb{R}(\widetilde{b})=\frac{b_{1}+b_{2}+b_{3}}{4} "$ Kumar et al. (2011)

"Assume that a stock has a one-week measurement horizon. The returns are computed from the preceding week $\left(t_{1}\right)$ to the end of the current week $(t)$. The geometric rate of return $r_{t}$ (logarithmic of the price) and geometric mean return $R_{t}$ for $t=1, \ldots, \mathrm{n}$ defined as follows": Philippe 2001)

$$
r_{t}=\ln \frac{P_{t}}{P_{t-1}} R_{t}=\frac{\sum_{i=1}^{n} t}{n}
$$

The standard deviation $(\sigma)$ is one of the most widely used risk measurements. Average squared deviation (variance) and The standard deviation on the $i^{\text {th }}$ asset respectively are given by; Rumpf (1990)

$$
\begin{gathered}
\sigma^{2}=\sum_{i=1}^{T} \frac{\left(r_{i t}-\bar{r}_{i}\right)^{2}}{T} \\
\sigma=\sqrt{\sum_{i=1}^{T} \frac{\left(r_{i t}-\bar{r}_{i}\right)^{2}}{T}}
\end{gathered}
$$


A diagonal variance is normally included in the variance-covariance matrix, with the covariance(CV) appearing in the off-diagonal member. The formula for determining the covariance of any two assets $i, j$ is calculated as follows(ALI et al. (2020)).

$$
C V\left(R_{i}, R_{j}\right)=\frac{1}{T} \sum_{i=1}^{T}\left(r_{i t}-\bar{r}_{i}\right)\left(r_{j t}-\bar{r}_{j}\right)
$$

\subsection{Genetic Algorithm (GA)}

(GA) is a heuristic method used in Artificial Intelligence and Mathematics to address optimization problems. Darwin's theory of evolution inspired GA based on an intelligent exploitation of a random search. This algorithm mimics natural selection, in which the fittest individuals are chosen for reproduction in order to create offspring the next generations. The selection of the fittest individuals from a population begins the natural selection process. They generate offspring which inherit the parents' qualities and are passed down to the next generation. If parents are active and fit, their children will be fitter than they are and have a better chance of surviving. This procedure will continue to iterate until a generation of the fittest individuals is discovered. Selection, crossover, mutation, and elitism are the fundamental GA operators. Binary tournament selection, simulated binary crossover (SBX), polynomial mutations, and elitism operators are used in the this paper 


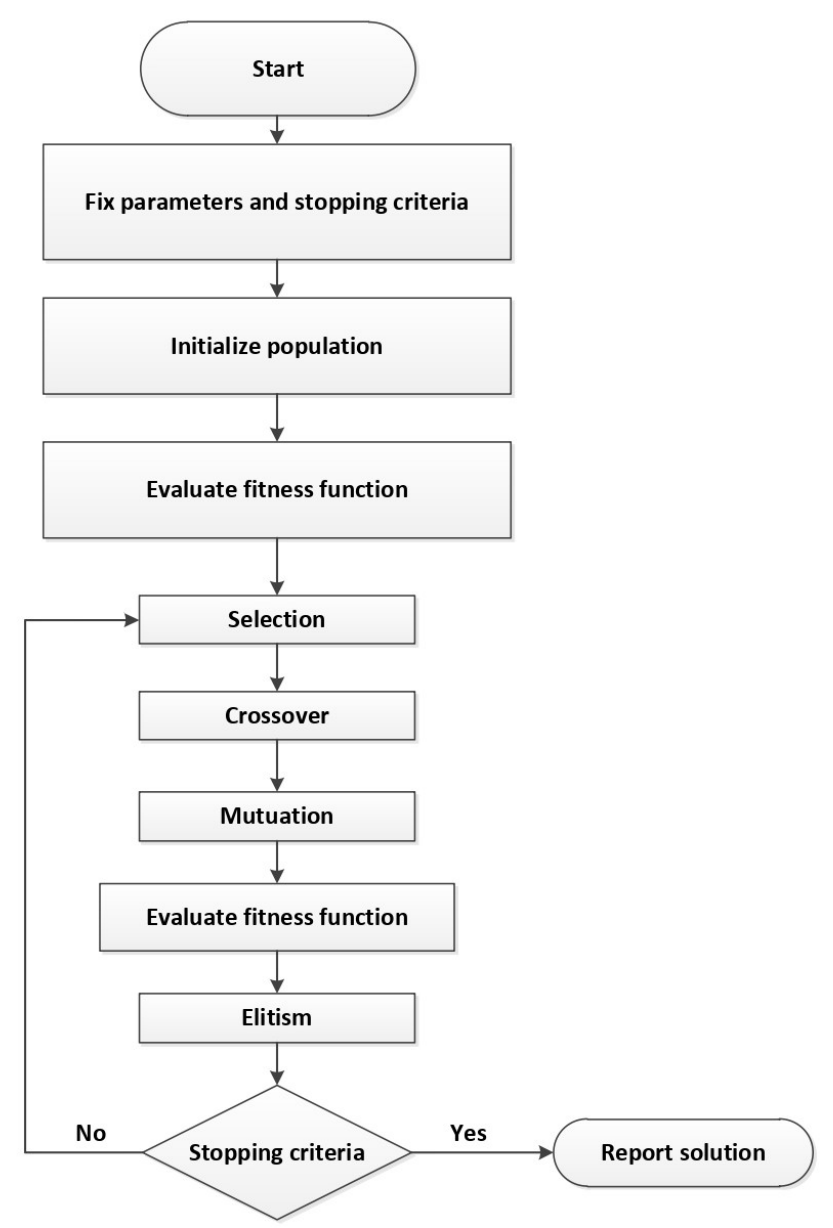

Figure 1: Flow chart of a genetic algorithm (GA).

\subsubsection{Termination}

If the population has converged, then algorithm will end, i.e. produces children who are not significantly different from the preceding generation. The genetic algorithm is thus considered to have generated a set of solutions to our problem.

\section{Mathematical Model}

Assume stocks $S_{1}, S_{2}, \ldots, S_{n}(n \geq 2)$ have random return. Let $\mu_{j} \sigma_{j}$, and $\widetilde{x_{j}}$ represent the geometric mean return of the stock $S_{j}$, the standard deviation of stock $S_{j}$, and the proportion of 
the entire fund invested in the portfolio (set of stock $\mathrm{j}$ ) respectively. For $i \neq j, \rho_{i j}$ denotes the correlation coefficient of stocks $S_{i}$ and $S_{j}$. Let $\widetilde{\mu}=\left[\widetilde{\mu_{1}}, \widetilde{\mu_{2}}, \ldots, \widetilde{\mu_{3}}\right]$. One can represent the expected fuzzy return and fuzzy variance of the resulting fuzzy portfolio $\tilde{x_{1}}, \tilde{x_{2}}, \ldots, \tilde{x_{n}}$ - as follows:

$$
\begin{array}{r}
\widetilde{E}[\widetilde{x}]=\widetilde{x_{1}} \widetilde{\mu_{1}}+\widetilde{x_{2}} \widetilde{\mu_{2}}+\ldots+\widetilde{x_{n}} \widetilde{\mu_{n}} \\
\widetilde{V}[\widetilde{x}]=\sum_{i, j} \rho_{i} j \widetilde{\sigma}_{i} \widetilde{\sigma}_{j} \widetilde{x_{i}} \widetilde{x_{j}}=\widetilde{x^{T}} \widetilde{H} \widetilde{x} \\
\rho_{i} j=1
\end{array}
$$

Mathematically, this formulation together with (3.1) produces a FQP problem for portfolio optimization:

$$
\widetilde{\min }: \frac{1}{2} \widetilde{x}^{T} \widetilde{H} \widetilde{x} \oplus \widetilde{c}^{T} \widetilde{x}
$$

Subject to

$$
\begin{gathered}
\widetilde{\mu}^{T} \widetilde{x} \geq \widetilde{R} \\
\sum_{i=1}^{n} \widetilde{x}_{i}=\widetilde{b}_{i} \\
\tilde{x}_{j} \geq \quad 0
\end{gathered}
$$

Where $\widetilde{x}^{T} \widetilde{H} \widetilde{x}$, is fuzzy variance.

$\widetilde{\mu}^{T} \widetilde{x}$, is fuzzy expected return, $\widetilde{R}$ is the least fuzzy target value of expected return and $\widetilde{b}_{i}$ is the sum of the fuzzy assets invested in each stocks.

$\widetilde{H} \widetilde{x} \geq 0$ (since variance is always non-negative and matrix resulted from all the core values of $\widetilde{H}$, is positive semi-definite.) Furthermore, because $\mathrm{H}$ is assumed to be semi-positive definite, the variance of the portfolio variables is a strictly convex function, and there is a single fuzzy portfolio $\widetilde{x} j$ with the minimum fuzzy variance. 


\section{GA based Method for FQP Problem with Fuzzy De- cision Variables}

The algorithmic stages for solving the FQP problem with fuzzy decisions variables are as follows:

Step 1 Fix the GA parameters as well as the termination criterion.

Step 2 The GA population is initialized using the constraints provided.

Step 3 Evaluate the fitness function.

Step 4 Select the fitter chromosome according to the fitness function result.

Step 5 Apply Crossover and Mutation respectively.

Step 6 Again calculate the fitness function.

Step 7 Apply Elitism.

Step 8 Examine the criteria for stopping. If the current population is achieved, the current population is the best population. Otherwise, go to step 4.

\section{Theoretical Case Study}

The crisp risk of selected and nominated to be risk adjusted return portfolio of five stocks(stock 1 , stock 2 , stock 3,stock 4,and stock 5), is converted in to triangular fuzzy risk of stock portfolio. Moreover we have converted the target value and the sum of all the individual fuzzy

asset of stocks respectively are $\widetilde{R}=(0.04,0.05,0.06)$ and $\sum_{j=1}^{m} \tilde{x}_{j}=(0.5,1,1.5)$, symbolizing each of the five stocks: $\widetilde{x_{1}}, \widetilde{x_{2}}, \widetilde{x_{3}}, \widetilde{x_{4}}$ and $\widetilde{x_{5}}$. The geometric mean and variance-covariance matrix have been transformed into fuzzy geometric mean and fuzzy variance-covariance matrix respectively. Equations from 2.2 to 2.4, are also used to determine the on and off diagonal 
elements of the variance-covariance matrix. Below are all of the fuzzy values that have been transformed:

$\widetilde{\mu}=[(0.0024,0.0025,0.0026),(-0.0025,-0.0024,-0.0023),(-0.0043,-0.0042,-0.0041)$, $(-0.0106,-0.0105,-0.0104),(0.0041,0.0042,0.0043)]$ 


$$
\widetilde{H}_{5 \times 5}=\left(\begin{array}{l}
(0.0047,0.0048,0.0049)(0.0006,0.0007,0.0008)(0.0015,0.0016,0.0017)(0.0010,0.0011,0.0012)(0.0020,0.0021,0.0022) \\
(0.0006,0.0007,0.0008)(0.0036,0.0037,0.0038)(0.0014,0.0015,0.0016)(0.0009,0.0010,0.0011)(0.0002,0.0003,0.0004) \\
(0.0015,0.0016,0.0017)(0.0014,0.0015,0.0016)(0.0034,0.0035,0.0036)(0.0012,0.0013,0.0014)(0.0012,0.0013,0.0014) \\
(0.0010,0.0011,0.0012)(0.0009,0.0010,0.0011)(0.0012,0.0013,0.0014)(0.0052,0.0053,0.0054)(0.0003,0.004,0.005) \\
(0.0020,0.0021,0.0022)(0.0002,0.0003,0.0004)(0.0012,0.0013,0.0014)(0.0003,0.0004,0.0005)(0.0036,0.0037,0.0038)
\end{array}\right)
$$


One can develop a mathematical FQP model as indicated in 3.1 to 3.4 utilizing the information supplied in case study 5 .

$\max : \tilde{Z}=(0.0047,0.0048,0.0049) \otimes \tilde{x}_{1}^{2} \oplus(0.0036,0.0037,0.0038) \otimes \tilde{x}_{2}^{2} \oplus(0.0034,0.0035,0.0036) \otimes \tilde{x}_{3}^{2} \oplus$

$(0.0052,0.0053,0.0054) \otimes \tilde{x}_{4}^{2} \oplus(0.0036,0.0037,0.0038) \otimes \tilde{x}_{5}^{2} \oplus 2(0.006,0.007,0.008) \tilde{x}_{1} \otimes \tilde{x}_{2} \oplus$

$2(0.0015,0.0016,0.0017) \otimes \tilde{x}_{1} \otimes \tilde{x}_{3} \oplus 2(0.0010,0.0011,0.0012) \otimes \tilde{x}_{1} \otimes \tilde{x}_{4} \oplus$

$2(0.008,0.009,0.001) \otimes \tilde{x}_{2} \otimes \tilde{x}_{4} \oplus 2(0.0002,0.0003,0.0004) \otimes \tilde{x}_{2} \otimes \tilde{x}_{5} \oplus$

$2(0.0012,0.0013,0.0014) \otimes \tilde{x}_{3} \otimes \tilde{x}_{4} \oplus 2(0.0013,0.0014,0.0015) \otimes \tilde{x}_{3} \otimes \tilde{x}_{5} \oplus$

$2(0.0003,0.0004,0.0005) \otimes \tilde{x}_{4} \otimes \tilde{x}_{5}$

Subject to

$(0.0024,0.0025,0.0026) \otimes \tilde{x}_{1} \oplus(-0.0025,-0.0024,-0.0023) \otimes \tilde{x}_{2} \oplus(-0.0043,-0.0042,-0.0041) \otimes \tilde{x}_{3}$

$$
\begin{gathered}
(-0.0106,-0.105,-0.0104) \otimes \tilde{x}_{4} \oplus(0.0041,0.0042,0.0043) \otimes \tilde{x}_{5} \geq(0.04,0.05,0.06) \\
\tilde{x}_{1} \oplus \tilde{x}_{2} \oplus \tilde{x}_{3} \oplus \tilde{x}_{4} \oplus \tilde{x}_{5}=(0.5,1,1.5) \\
0 \leq x_{1} \leq x_{2} \leq x_{3} 0 \leq x_{4} \leq x_{5} \leq x_{6} \\
0 \leq x_{7} \leq x_{8} \leq x_{9} \\
0 \leq x_{10} \leq x_{11} \leq x_{12} \\
0 \leq x_{13} \leq x_{14} \leq x_{15}
\end{gathered}
$$

Note: Variables; $\tilde{x}_{1}=\left(x_{1}, x_{2}, x_{3}\right), \tilde{x}_{2}=\left(x_{4}, x_{5}, x_{6}\right), \tilde{x}_{3}=\left(x_{7}, x_{8}, x_{9}\right), \tilde{x}_{4}=\left(x_{10}, x_{11}, x_{12}\right)$ and $\tilde{x}_{5}=\left(x_{13}, x_{14}, x_{15}\right)$ are assumed to be triangular fuzzy decision variables. 


\section{Results of Simulation}

\subsection{Parameters of the algorithm}

The proposed GA based ranking function approach is coded in MATLAB. The total number of iterations(T) is assumed to be 100 and 150. Using more than ten simulations, the fuzzy optimal solutions are documented. All the the fuzzy solutions are reported as to four decimal places. $P_{c}=0.9$ and $P_{m}$ respectively are probability of $\operatorname{crossover}\left(P_{c}\right)$ and probability of mutation $\left(P_{m}=\frac{1}{V}\right.$, where $\mathrm{V}$ is number real variables in a triangular fuzzy decision variable). This probabilities were used in the extensive study along with the population size of 100 .

\subsection{Result and Discussion: FQP problem using GA}

The FQP problem has been solved using GA in this research without the need to find a deterministic equivalent. A triangular fuzzy number is considered to represent both coefficients and non-negative decision variables. One of the most significant advantages of the approach is that it eliminates the need to find a deterministic equivalent to solve the problem. Another advantage is, because the current world's realistic assumptions of parameters and decision factors are not accurate, the programming problem enables decision maker to realistically decide the decision. The case study(5) results show that the above approach is quite effective. In this study, most redundant approximated optimal values are used as minimized risks(objective functional values). The tables; 1 and 2 respectively recapitulate fuzzy asset allocation and the corresponding fuzzy optimal risk values of risk adjusted stock portfolio as follows: 
Table 1: optimal solutions for $\mathrm{T}=100$

\begin{tabular}{|c|c|c|c||c|c|c|}
\hline Simulation & $\widetilde{Z}$ & $\tilde{x}_{1}$ & $\tilde{x}_{2}$ & $\tilde{x}_{3}$ & $\tilde{x}_{5}$ \\
\hline 1 & $(0.0003,0.0144,0.0150)$ & $(0.2946,0.3496,0.3712)$ & $(0.0333,0.1104,0.3942)$ & $(0.1811,0.2274,0.2989)$ & $(0.0108,0.0167,0.8185)$ & $(0.0644,0.0636,0.0234)$ \\
\hline 2 & $(0.0016,0.0137,0.0524)$ & $(0.3171,0.4323,0.7664)$ & $(0.1271,0.0664,0.5735)$ & $(0.2063,0.6715,0.9217)$ & $(0.0440,0.8397,0.0079)$ & $(0.0079,0.0837,0.0911)$ \\
\hline 3 & $(0.0003,0.01393,0.02389)$ & $(0.0058,0.1781,0.4661)$ & $(0.0665,0.2331,0.4646)$ & $(0.2449,0.2963,0.4115)$ & $(0.01670,0.0937,0.0232)$ & $(0.0434,0.0098,0.1310)$ \\
\hline 4 & $(0,0.0196,0.0138)$ & $(0.0942,0.1482,0.4481)$ & $(0.0131,0.0808,0.3046)$ & $(0.03260 .64760 .6975)$ & $(0.0095,0.0394,0.5458)$ & $(0.010270 .06530 .01187)$ \\
\hline 5 & $(0.0010,0.0138,0.0219)$ & $(0.1797,0.0577,0.8105)$ & $(0.1180,0.1384,0.0455)$ & $(0.21894,0.4027,0.52034)$ & $(0.0471,0.1220,0.0363)$ & $(0.0830,0.0507,0.0967)$ \\
\hline
\end{tabular}

Table 2: optimal solutions for $\mathrm{T}=150$

\begin{tabular}{|c|c|c|c|c|c|c|}
\hline Simulation & $\widetilde{Z}$ & $\widetilde{x}_{1}$ & $\widetilde{x}_{2}$ & $\widetilde{x}_{3}$ & $\widetilde{x}_{4}$ & $\widetilde{x}_{5}$ \\
\hline 1 & $(0.0020,0.0133,0.0163)$ & $(0.0205,0.0091,0.0478)$ & $(0.6520,0.7067,0.8308)$ & $(0.1108,0.0655,0.3583)$ & $(0.0108,0.0167,0.8185)$ & $(0.0015,0.0927,0.8695)$ \\
\hline 2 & $(0.0016,0.0135,0.0041)$ & $(0.1241,0.0322,0.0022)$ & $(0.1271,0.0664,0.5735)$ & $(0.0185,0.0320,0.0685)$ & $(0.1456,0.2376,0.4410)$ & $(0.1114,0.4198,0.0533)$ \\
\hline 3 & $(0.0020,0.0138,0.0163)$ & $(0.0978,0.2514,0.3576)$ & $(0.1593,0.0120,0.68663)$ & $(0.2709,0.2962,0.47032)$ & $(0.0065,0.0178,0.3184)$ & $(0.0232,0.1559,0.0596)$ \\
\hline 4 & $(0.0008,0.0137,0.0125)$ & $(0.08703,0.0167,0.0598)$ & $(0.0038,0.0100,0.4967)$ & $(0.3643,0.8233,0.9312)$ & $(0.0182,0.0012,0.4161)$ & $(0.1258,0.0405,0.0134)$ \\
\hline 5 & $(0.0002,0.0137,0.0074)$ & $(0.10356,0.1345,0.3770)$ & $(0.0714,0.1477,0.1904)$ & $(0.0069,0.0670,0.1139)$ & $(0.0727,0.2340,0.6314)$ & $(0.0727,0.2340,0.6314)$ \\
\hline
\end{tabular}




\section{Conclusion}

In this work, a solution strategy for fuzzy quadratic programming(FQP) problem with fuzzy decision variables is presented, where fuzzy risks(variance) of each stocks portfolio considered as objectives function and the fuzzy constraints are considered to be fuzzy expected return and fuzzy total assets of stock portfolio. We focussed on five stocks that were chosen based on a number of factors, including beta $(\beta)$, Treynor ratio (M-squared test) and variance. Crisp risk, which is variance, and crisp constraints of the programming problem are converted into fully fuzzy, where both the objective and constraint parameters are triangular fuzzy number. Decision variables are assumed to be triangular fuzzy variable. We considered two iterations, $\mathrm{T}=100$ and $\mathrm{T}=150$, and found that both produced nearly the same result, indicating that the algorithm had terminated. Popular popular non-traditional search techniques; namely, particle swarm optimization (PSO), ant colony optimization (ACO) and simulated annealing (SA) could be utilized To identify the optimal solutions to the programming problem.

Acknowledgements We, the authors, would like to express our gratitude to the researchers whose findings we have mentioned in this study.

Funding No funding has been done in this paper.

Data availability We took crisp data from work of MUSSAFI \& ISMAIL (2021) and changed it into triangular fuzzy number. Moreover in the submitted manuscript, all the models developed or used during the study paper are included.

\section{Declarations}

Conflict of interest There is no conflict of interest by the authors with anyone whosoever is connected with this research. 


\section{References}

ALI, H., HAO, Y. \& AIJUAN, C. (2020), 'Innovation capabilities and small and medium enterprises' performance: An exploratory study', The Journal of Asian Finance, Economics, and Business 7(10), 959-968.

Bellman, R. E. \& Zadeh, L. A. (1970), 'Decision-making in a fuzzy environment', Management science $\mathbf{1 7}(4), \mathrm{B}-141$.

Fathy, E. (2019), 'A modified fuzzy approach for the fully fuzzy multi-objective and multilevel integer quadratic programming problems based on a decomposition technique', Journal of Intelligent 8 Fuzzy Systems 37(2), 2727-2739.

Gessesse, A. A., Mishra, R., Acharya, M. M. \& Das, K. N. (2020), 'Genetic algorithm based fuzzy programming approach for multi-objective linear fractional stochastic transportation problem involving four-parameter burr distribution', International Journal of System Assurance Engineering and Management 11(1), 93-109.

Goldfarb, D. \& Idnani, A. (1983), 'A numerically stable dual method for solving strictly convex quadratic programs', Mathematical programming 27(1), 1-33.

Kumar, A., Kaur, J. \& Singh, P. (2011), 'A new method for solving fully fuzzy linear programming problems', Applied mathematical modelling 35(2), 817-823.

Lawson, C. L. \& Hanson, R. J. (1995), Solving least squares problems, SIAM.

Mussafi, N. S. M. \& Ismail, Z. (2020), Quadratic programming for optimizing the diversified shariah stock portfolio, in 'The 2nd International Seminar on Science and Technology (ISSTEC 2019)', Atlantis Press, pp. 139-147.

MUSSAFI, N. S. M. \& ISMAIL, Z. (2021), 'Optimum risk-adjusted islamic stock portfolio using the quadratic programming model: An empirical study in indonesia', The Journal of Asian Finance, Economics and Business 8(5), 839-850. 
Nasseri, H., Baghban, A. \& Mahdavi, I. (2019), 'A new approach for solving fuzzy multiobjective quadratic programming of water resource allocation problem', Journal of Industrial Engineering and Management Studies 6(2), 78-102.

Philippe, J. (2001), 'Value at risk: the new benchmark for managing financial risk', $N Y$ : McGraw-Hill Professional .

Powell, M. J. D. (1985), On the quadratic programming algorithm of goldfarb and idnani, in 'Mathematical Programming Essays in Honor of George B. Dantzig Part II', Springer, pp. $46-61$.

Pramanik, S. \& Dey, P. P. (2011), 'Multi-objective quadratic programming problem: a priority based fuzzy goal programming', international journal of computer Applications 26(10), 30-35.

Rout, P. K., Nanda, S. \& Acharya, S. (2019), 'Multi-objective fuzzy probabilistic quadratic programming problem', International Journal of Operational Research 34(3), 387-408.

Rumpf, H. (1990), 'The characteristics of systems and their changes of state disperse', Particle Technology, Chapman and Hall; Springer: Berlin/Heidelberg, Germany pp. 8-54. 